\title{
Spread of Clostridium botulinum in the soils of Georgia
}

\author{
Ekaterine Zhorzholiani ${ }^{\star}$, Neli Chakvetadze, Guram Katsitadze and Paata Imnadze
}

National Center for Disease Control and Public Health, Tbilisi, Georgia

\section{Objective}

The main focus of this study was to study the spread of botulism in Georgia and the biological characteristics of the strains of Clostridium botulinum isolated from territories in the country.

\section{Introduction}

Accumulation of $C$. botulinum in soil occurs through excretion of bacterial spores from the intestines of humans, animals, birds and fish. In Georgia, during the winter season, the population consumes homemade vegetable preserves, which are made of locally produced (as well as imported) vegetables. Historical surveys confirmed that the presence of $C$. botulinum in the soil is widespread. Some researchers consider C. botulinum a characteristic component of soil flora.

\section{Methods}

Soil samples were collected from areas, where from 2001-2002 cases of botulism caused by homemade vegetable preserves (produced from vegetables cultivated in those areas) were registered. Soil samples were collected from Kakheti, Shida Kartli, Kvemo Kartli, Samtkhe-Javakheti, and Samegrelo regions. Standard bacteriology and PCR were used to confirm the presence of C. botulinum from soil samples. Separation of strains and their examination was conducted in accordance with the scheme provided by the CDC Atlanta Reference Laboratory (USA), which was later tested by NCDC. Toxigenicity and toxin production of strains were tested using a biotest on white mice.

\section{Results}

In total, 258 soil samples were tested, from which, 40 (15.5\%) cultures of $C$. botulinum type B were obtained. Toxigenicity and toxin production were confirmed through biotests. These results confirm the presence of $C$. botulinum in agricultural lands, which causes contamination of vegetables cultivated on those lands, which are used for the preparation of homemade preserves, causing botulism in humans.

\section{Conclusions}

For the purpose of finding solutions to botulism, it is essential to verify the ecology of the pathogen through establishing the prevalence of bacteria in different soil types. It was shown that some areas of Georgia, where vegetable growing is greatly developed, and which, are the main sources of crops, are highly contaminated with C. botulinum. In Georgia, land used for agriculture is contaminated with C. botulinum. C. botulinum type B was isolated from 40 cultures obtained from 258 soil samples, which represents contamination in $15.5 \%$ of sampled areas. These results suggest that vegetables and melons may be highly contaminated as well. All cases of $C$. botulinum in humans that were researched were connected to homemade canned vegetables.

\section{Keywords}

C. botulinum; Soil; Spores; Strains

\section{Acknowledgments}

This work is supported by the National Botulism Laboratory Team, and CDC Atlanta, Georgia. Participation in this conference was made possible by financial support provided by the US Defense Threat Reduction Agency. The findings, opinions and views expressed herein belong to the authors and do not reflect an official position of the Department of the Army, Department of Defense, or the US Government, or any other organization listed.

\section{*Ekaterine Zhorzholiani}

E-mail: Eka.Zhorzholiani@ch2m.com 